\title{
An Ellipsometric Study of Protein Adsorption at the Saliva-Air Interface
}

\author{
H. J. HOLTERMAN, ${ }^{*, 1}$ E. J. 'S-GRAVENMADE, $\dagger$ H. A. WATERMAN, $*$ \\ J. MELLEMA, ${ }^{*}$ AND C. BLOM* \\ *Rheology Group, Faculty of Applied Physics, University of Twente, Enschede, The Netherlands; and $\dagger$ Laboratory \\ for Materia Technica, University of Groningen, Groningen, The Netherlands
}

Received February 12, 1988; accepted May 24, 1988

\begin{abstract}
At the liquid-air interface of human saliva a protein layer is adsorbed. From ellipsometric measurements it was found that the thickness of the surface layer ranged from 400 to $3600 \AA$ and the amount of protein material adsorbed was $9-340 \mathrm{mg} / \mathrm{m}^{2}$. Based on the concentration of protein in the layer the samples could be classified into two groups: a low concentration ( ca. $0.15 \mathrm{~g} / \mathrm{ml}$ ) and a high concentration $(0.7-$ $1.1 \mathrm{~g} / \mathrm{ml})$. In the low concentration group the surface layers appeared to be thin $(500-600 \AA)$ while those in the high concentration group appeared to be much thicker (1000-3500 $\AA$ ). A correlation between the bulk pH and the thickness of the surface layer could be established. (C) 1989 Academic Press, Inc.
\end{abstract}

\section{INTRODUCTION}

The physiological importance of saliva in humans becomes obvious when saliva is secreted to a too small (hyposalivation) extent. The reduced salivary secretion results in a wide scale of complaints, such as a dry and burning sensation of the mouth and difficulties in chewing, swallowing, speaking, and sleeping. To combat these complaints, the lack of saliva has to be compensated. For those patients in which salivary flow cannot be stimulated or only insufficiently, a saliva substitute may be an alternative. To produce a saliva substitute having essentially the same properties as human saliva with respect to its functions, a thorough study of the properties of human saliva is necessary. Preliminary measurements showed the existence of a rigid layer of adsorbed protein at the air-liquid interface (1). This layer forms rapidly and achieves a remarkable thickness (compared to protein layers described in literature). The establishment of the characterization of such a layer may be helpful in assessing potential saliva substitutes. This study deals with the time dependence of layer thickness and refractive index during

${ }^{1}$ To whom correspondence should be addressed. protein adsorption at the air-liquid interface of submandibular, parotid, and whole saliva using ellipsometry.

\section{THEORY OF ELLIPSOMETRY}

The state of polarization of a plane coherent beam of light can be characterized by the amplitude ratio $\left|E_{\mathrm{p}}\right| /\left|E_{\mathrm{s}}\right|$ and phase difference $\delta_{\mathrm{p}}-\delta_{\mathrm{s}}$ of the components of the electric field vector parallel $\left(E_{\mathrm{p}}=\left|E_{\mathrm{p}}\right| \exp i \delta_{\mathrm{p}}\right)$ and perpendicular $\left(E_{\mathrm{s}}=\left|E_{\mathrm{s}}\right| \exp i \delta_{\mathrm{s}}\right)$ to the plane of incidence. After reflection by an interface these components can be described by $E_{\mathrm{p}}^{\mathrm{r}}=R_{\mathrm{p}}$. $E_{\mathrm{p}}^{\mathrm{i}}$ and $E_{\mathrm{s}}^{\mathrm{r}}=R_{\mathrm{s}} \cdot E_{\mathrm{s}}^{\mathrm{i}}$, where $R_{\mathrm{p}}$ and $R_{\mathrm{s}}$ are the complex overall reflection coefficients for the two components. The superscripts $i$ and $r$ refer to incident and reflected beams, respectively. The change of polarization after reflection is described using

$$
\rho=\frac{R_{\mathrm{p}}}{R_{\mathrm{s}}} \equiv \tan \psi \cdot \exp i \Delta,
$$

where $\psi$ and $\Delta$ are real quantities, defined for convenience by the latter equality.

From Eq. [1] it follows that

$$
\tan \psi=\frac{\left|E_{\mathrm{p}}^{\mathrm{r}}\right| /\left|E_{\mathrm{s}}^{\mathrm{r}}\right|}{\left|E_{\mathrm{p}}^{\mathrm{i}}\right| /\left|E_{\mathrm{s}}^{\mathrm{i}}\right|}
$$


and

$$
\Delta=\left(\delta_{\mathrm{p}}^{\mathrm{r}}-\delta_{\mathrm{s}}^{\mathrm{r}}\right)-\left(\delta_{\mathrm{p}}^{\mathrm{i}}-\delta_{\mathrm{s}}^{\mathrm{i}}\right),
$$

describing the change of the amplitude ratio and phase difference for the parallel and perpendicular components, respectively.

In our experimental setup, described in Section $3.2, \psi$ and $\Delta$ are determined from azimuthal angle measurements of the polarizer and analyzer at minimal transmission, i.e.,

$$
\begin{aligned}
& \psi=A \\
& \Delta=2 P+\pi / 2
\end{aligned}
$$

where $P$ and $A$ are the polarizer and analyzer extinction settings, respectively (2).

Combining Eqs. [1] and [4] the value of $P$ can be calculated from the measurements of $P$ and $A$.

From multiple reflections and refractions at the air-liquid interface the complex overall reflection coefficients $R_{\mathrm{p}}$ and $R_{\mathrm{s}}$ can be calculated by

$$
R_{j}=\frac{r_{01, j}+r_{12, j} \exp (-i \phi)}{1+r_{01, j} r_{12, j} \exp (-i \phi)},
$$

where $j$ represents either subscript $\mathrm{p}$ or subscript $s ; r_{01}$ and $r_{12}$ are the Fresnel reflection coefficients at the air (0)-film (1) interface and the film (1)-liquid (2) interface, respectively (3) (see also Fig. 1). The quantity $\phi$ represents the phase difference between two consecutive

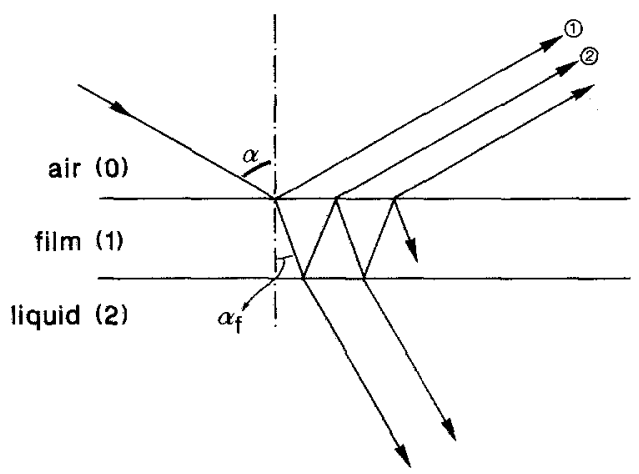

FIG. 1. Reflection and refraction rays at the interface of two media separated by a thin film. reflected rays (e.g., rays 1 and 2 in Fig. 1) and is given by

$$
\begin{aligned}
\phi & =\frac{4 \pi d n_{\mathrm{f}}}{\lambda} \cos \alpha_{\mathrm{f}} \\
& =\frac{4 \pi d}{\lambda} \sqrt{n_{\mathrm{f}}^{2}-n_{0}^{2} \sin ^{2} \alpha}
\end{aligned}
$$

in which Snell's law has been used to obtain the last equality; furthermore, $d$ is the film thickness; $\lambda$ is the wavelength of light in vacuum, $n_{0}$ is the refractive index of the medium above the film (i.e., air), $n_{\mathrm{f}}$ is the film refractive index, and $\alpha, \alpha_{\mathrm{f}}$ are angles of incidence and refraction, respectively. Combining Eqs. [1], [5], and [6], we can express $\rho$ in the Fresnel reflection coefficients and the phase difference $\phi$.

Apparently these equations determine implicitly $d$ and $n_{\mathrm{f}}$ if the other quantities are known.

\section{METHODS}

\subsection{Determination of $d n / d c$}

Since the protein content of saliva is too low (approximately $0.5 \mathrm{wt} \%$ (4)) to determine $d n / d c$ of saliva sufficiently accurately, we used a solution of bovine submaxillary mucin (BSM; Sigma M4503) instead, which is much like the proteins occurring in human saliva.

An aqueous solution of BSM (20.1 \pm 0.1 $\mathrm{mg} / \mathrm{ml}$ ) was diluted stepwise. After each dilution step the solution was homogenized by gentle stirring. At each stage the refractive index $n$ was measured. Since in the experiments the densities of all stages were approximately equal the relative concentration decrease $c_{k+1}$ / $c_{k}$ could be determined by weighing the solution before and after dilution,

$$
\frac{c_{k+1}}{c_{k}} \approx \frac{m_{k}}{m_{k}+\Delta m_{k}}
$$

where $k$ is the stage number, $c_{k}$ is the concentration at stage $k, m_{k}$ is the solution mass at concentration $c_{k}$, and $\Delta m_{k}$ is the mass of added water. 


\subsection{The Ellipsometer}

The experimental setup of the ellipsometer is shown in Fig. 2. From left to right the following devices are mounted:

1. He-Ne laser (Hughes: $3222 \mathrm{H}-\mathrm{PC}$ ), wavelength $\lambda=6328 \AA, 2 \mathrm{~mW}$;

2. quarter-wave plate QWP1, the fast axis at about $45^{\circ}$ to the plane of polarization of the laser light;

3. polarizer POL (Halle: PGL10), Glan prism type, mounted on a rotatable device which can be read to $0.01^{\circ}$ (setting value $P$ );

4. quarter-wave plate QWP2, identical to QWP1, fast axis at $-45.0^{\circ}\left( \pm 0.2^{\circ}\right)$ to the plane of incidence (setting value $Q$ );

5. sample trough, vertically adjustable, inner dimensions $65 \times 65 \times 6 \mathrm{~mm}^{3}$ approximately, temperature kept constant by means of a thermostat;

6. analyzer ANA, type and mounting identical to the polarizer (setting value $A$ );

7. Si-photodiode detector (EG\&G: UV100-BG), passing a simple amplifier unit the intensity is read from a digital multimeter.

The settings of POL, ANA, and QWP2 are all measured counterclockwise from the plane of incidence looking into the laser beam. The measuring technique has been described by several authors $(2,5)$ and is based on the extinction setting of the polarizer and analyzer.

The calculation of $d$ and $n_{\mathrm{f}}$ is as follows. As a start a value of $n_{f}$ is guessed, from which the Fresnel reflection coefficients are calculated. Knowing $\rho$ from the measurement of $P$ and $A$, the phase angle $\phi$ is calculated (Eqs. [1]

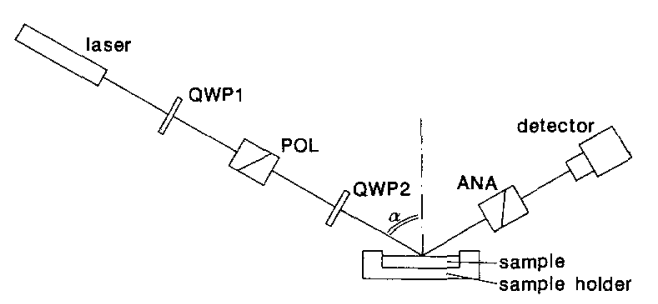

FIG. 2. Principle of the ellipsometer technique. and [5]), and from Eq. [6] the layer thickness $d$ is calculated. Generally the value of $d$ is a complex quantity, the imaginary part of which will vanish if the correct $n_{\mathrm{f}}$ value has been chosen. Thus a solution for $d$ and $n_{\mathrm{f}}$ is found by an iterative procedure. A detailed description of the numerical procedure is given by $\mathrm{Mc}$ Crackin (6).

Using the calculated $d$ and $n_{\mathrm{f}}$ values the total amount of adsorbed material per unit surface area is given by

$$
\Gamma=\frac{d \cdot\left(n_{\mathrm{f}}-n_{\mathrm{solv}}\right)}{d n / d c},
$$

where $n_{\text {solv }}$ is the refractive index of the solvent and $d n / d c$ is the refractive increment of the solution. This equation can only be used when $d n / d c$ can be considered as constant.

A FORTRAN program (6) was used both to calculate $n_{\mathrm{f}}$ and $d$ from $P$ and $A$ settings and to carry out several error-checking calculations. For the chosen adjustment ( $Q$ $=-45.0^{\circ}$ ) there are eight polarizer and analyzer extinction settings; they can be divided into two different groups or zones. It has been reported $(3,5)$ that the accuracy will increase when measurements are performed in different zones. In most cases, however, measurements in more than one zone would involve too much time to consider the surface layer as constant during the measurements, therefore in this study measurements were performed in one zone only.

Measurements at the surface of demineralized water were used to determine the angle of incidence $\alpha$ since $A_{0}$ (i.e., $A$ at $d=0$ ) is dependent on $\alpha$. In that situation $P_{0}$ (i.e., $P$ at $d=0$ ) should be equal to $45^{\circ}$ (in a particular zone). In this way $\alpha$ could be calculated from $A_{0}$ within $0.01^{\circ}$. In practice $P_{0}$ turned out to differ a few degrees from $45^{\circ}$, probably because of a very thin surface layer caused by small traces of pollution. However, such a very thin layer causes no significant deviation from $A_{0}$ and therefore it is not affecting the determination of $\alpha$. The setting error of QWP2 $\left(0.2^{\circ}\right)$ not only affects $A$ and $P$ readings but also affects the determination of $\alpha$. Especially in the 
case of a thin surface layer, thickness and refractive index appear to be very sensitive to this error, but the corresponding error in $\Gamma$ turns out to disappear almost completely, due to cancellation of systematic errors.

For a given $d$ and $n_{\mathrm{f}}$ value the $P$ and $A$ settings can be calculated. The settings plotted for different layer thicknesses at a constant refractive index result in a curve shown in Fig. 3.

Adsorption starts at $\left(P_{0}, A_{0}\right)$ where $d=0$. When $d$ increases at constant refractive index, $(P, A)$ follows the curve corresponding to that particular refractive index. When $d$ reaches the so-called critical thickness $d_{\mathrm{c}},(P, A)$ has returned to $\left(P_{0}, A_{0}\right)$. Further increase in thickness leads to a second walk of $(P, A)$ along the same curve (provided there is no absorption of light in the film). Therefore interpreting measurements of $P$ and $A$ will always include a periodicity of $d_{\mathrm{c}}$ in the layer thickness. This critical thickness corresponds to the phase difference $\phi$ reaching $2 \pi$. From Eq. [6] it follows that

$$
d_{\mathrm{c}}=\frac{\lambda / 2}{\sqrt{n_{\mathrm{f}}^{2}-n_{0}^{2} \sin ^{2} \alpha}} .
$$

The closed curve in Fig. 3 corresponds to a certain $n_{\mathrm{f}}$ value. Other $n_{\mathrm{f}}$ values will give other curves, however, all starting at $\left(P_{0}, A_{0}\right)$ since there is no physical difference at a zero thickness. Therefore small errors in $P$ and $A$ settings give rise to large errors in $d$ and $n_{\mathrm{f}}$ values at $(P, A)$ values close to $\left(P_{0}, A_{0}\right)$.

Choosing the angle of incidence $\alpha$ close to Brewster's angle $\theta_{\mathrm{B}}$ of the underlying bulk liquid, the calculated $(P, A)$ curves in Fig. 3 blow up. In that case the errors in $d$ and $n_{\mathrm{f}}$ due to errors in $P$ and $A$ decrease. However, reproducibility in $P$ and $A$ readings appears to decrease rapidly at $\alpha$ very close to $\theta_{\mathrm{B}}$, resulting in a complete loss of significance of calculated $d$ and $n_{\mathrm{f}}$ values. In the experiments an optimum was reached at $\left|\alpha-\theta_{\mathrm{B}}\right|$ between 0.5 and $1.0^{\circ}$ approximately.

Since the calculated $d$ and $n_{\mathrm{f}}$ values are not deduced independently, the error in $\Gamma$ appears to be less than that due to a propagation of errors attributed to $d$ and $n_{\mathrm{f}}$ values determined independently.

\subsection{Bulk Measurements}

To characterize the bulk liquid of each sample the following quantities were measured:

1. acidity, $\mathrm{pH}$ (Metrohm Herisan E603

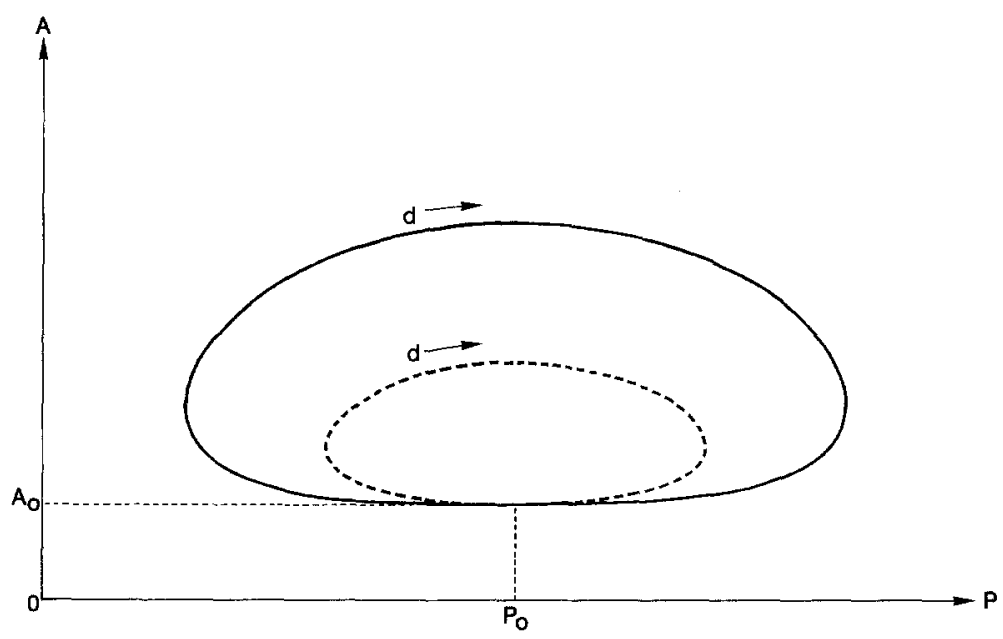

FIG. 3. Schematic representation of $P$ and $A$ at constant $n_{\mathrm{f}}$ and varying $d$. The broken curve corresponds to a different $n_{\mathrm{f}}$ value. 
pH meter; probe: Broadley-James-Corp. type 9111);

2. density, $\rho$ (Paar DMA 40 digital density meter);

3. refractive index, $n_{\mathrm{s}}$ (Abbe refractometer);

4. electrical conductivity, $\Lambda$ (Radiometer CDM2d conductivity meter; probe: Radiometer PP1042).

All measurements were carried out at $25^{\circ} \mathrm{C}$ approximately.

\section{MATERIALS}

Three types of human saliva were studied: submandibular, parotid, and whole saliva. A total of 22 saliva samples from five healthy males were used. Saliva secretion was started at about one hour after breakfast and was stimulated by a citric acid solution dipped frequently onto the tongue in the case of submandibular and parotid saliva. In the case of whole saliva secretion no such stimulus could be used and therefore secretion was stimulated by chewing a piece of parafilm. Before starting the saliva collection the mouth was rinsed with water. The first 1-3 ml of each sample was not used in the experiments. About $40 \mathrm{ml}$ was needed of each sample to perform a series of different experiments using the same saliva sample, of which $10 \mathrm{ml}$ was used to fill the sample holder in the ellipsometer. Submandibular saliva was collected using a modified Schneyer's apparatus (7). Parotid saliva was collected using a set of modified Lashley's cups (8). The reservoir was filled within half an hour after secretion had finished. After filling the reservoir the surface was wiped by means of a bar moving across the surface to create a fresh air-liquid interface. The actual measurements were started immediately after that. In this way the first measurement data were obtained within approximately $3 \mathrm{~min}$ after wiping the surface. All measurements were performed at $25^{\circ} \mathrm{C}$. Submandibular and in particular whole saliva samples were more viscous than parotid saliva samples and behaved in some cases even quite elastically. The adsorbed surface layers were in most cases visible and appeared to be very vulnerable.

\section{EXPERIMENTS}

\subsection{Determination of $d n / d c$}

The results shown in Fig. 4 reveal within the measured range a linear relationship from which

$$
d n / d c=0.17( \pm 0.01) \mathrm{ml} / \mathrm{g} .
$$

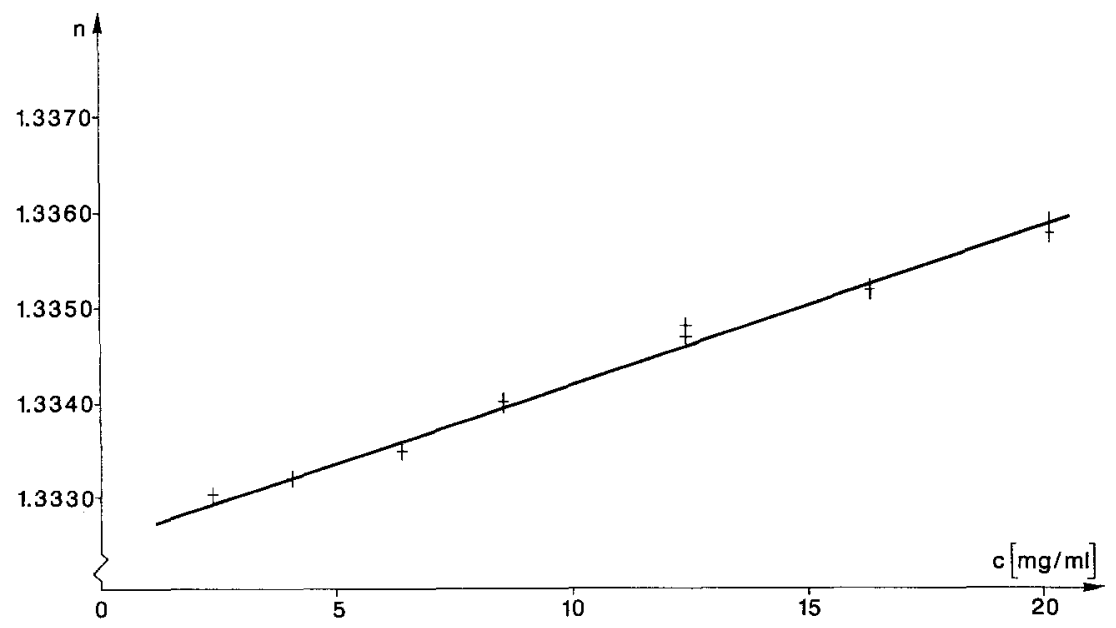

FIG. 4. Bulk refractive index of a BSM solution as a function of concentration. 


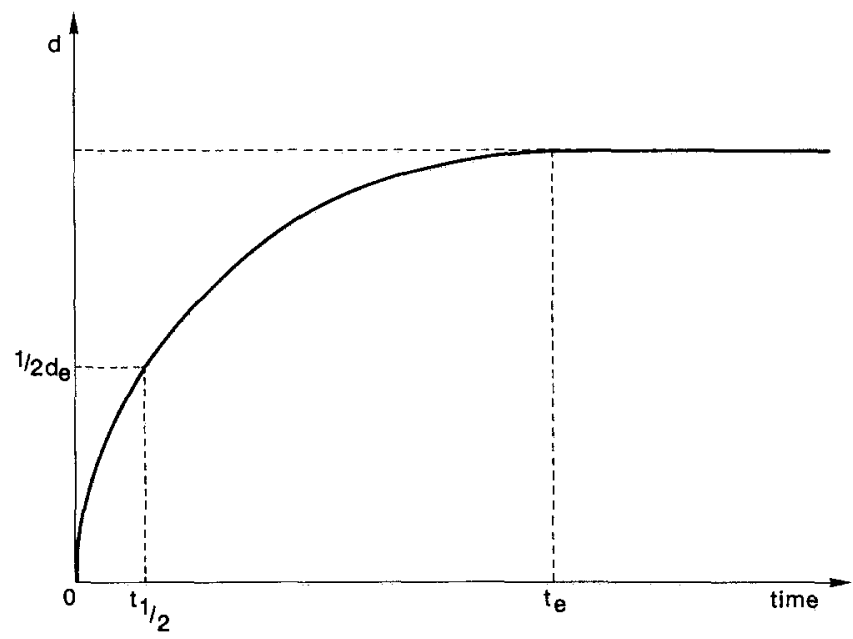

FIG, 5. Layer thickness as a function of time. End value $d_{\mathrm{e}}$ was reached at about time $t_{\mathrm{e}}$, while at time $t_{1 / 2}$ a thickness of about $d_{\mathrm{e}} / 2$ was reached.

The concentrations appearing in the surface layers are much higher (up to about 50 times higher) than the starting concentration used here. The value of $d n / d c$ at high concentrations may not be equal to that at low concentrations. At low concentrations up to about $0.35 \mathrm{~g} / \mathrm{ml}$, however, the rate $d n / d c$ appears to be relatively constant even among different proteins (2). Although the exact relationship between $n$ and $c$ at higher concentrations is not known, in the present study $d n / d c$ is assumed to be constant up to at least the concentrations occurring in the adsorbed protein layers.

\subsection{Ellipsometric Measurements}

Ellipsometric measurements were performed during approximately 4 to $5 \mathrm{~h}$. In most cases the calculated refractive index $n_{\mathrm{f}}$ increased very rapidly and became constant within one hour while the layer thickness $d$ revealed an adsorption curve as shown in Fig. 5. Though almost all adsorption curves showed similar graphs, the thickness end values $d_{\mathrm{e}}$ appeared to vary from 400 up to about $3600 \AA$. In most cases $t_{\mathrm{e}}$ was 3 to $4 \mathrm{~h}$, while $t_{1 / 2}$ was reached within half an hour.

The amount of adsorbed material per unit

TABLE I

Measured Bulk Properties of the Saliva Samples

\begin{tabular}{|c|c|c|c|c|c|c|}
\hline & \multicolumn{2}{|c|}{ SUB $(\# 8)$} & \multicolumn{2}{|c|}{ PAR (\#7) } & \multicolumn{2}{|c|}{ WHL (\#7) } \\
\hline & Mean & Range & Mean & Range & Mean & Range \\
\hline $\mathrm{pH}$ & 7.4 & $6.8-7.9$ & 7.9 & $6.8-8.4$ & 7.5 & $7.4-7.7$ \\
\hline$\rho\left(\mathrm{kg} / \mathrm{m}^{3}\right)$ & 999.3 & $998.5-1000.1$ & 1010.5 & $998.5-1001.9$ & 999.3 & $999.0-1000.0$ \\
\hline$n_{\mathrm{s}}$ & 1.3333 & $1.3330-1.3335$ & 1.3338 & $1.3333-1.3340$ & 1.3333 & $1.3332-1.3335$ \\
\hline$\Lambda\left(10^{-3} \Omega^{-1} \mathrm{~cm}^{-1}\right)$ & 3.6 & $2.4-6.0$ & 5.1 & $3.0-7.0$ & 3.3 & $2.9-4.0$ \\
\hline \multicolumn{7}{|l|}{ Flow rate } \\
\hline$(\mathrm{ml} / \mathrm{min})$ & 0.9 & $0.6-1.1$ & 1.0 & $0.3-1.9$ & 0.7 & $0.3-0.9$ \\
\hline
\end{tabular}

Note. Abbreviations: SUB, submandibular; PAR, parotid; WHL, whole; \#, number of samples. 
TABLE II

Classification Scheme Based on $c_{\mathrm{f}}$ Values

\begin{tabular}{|c|c|c|c|c|c|c|c|c|}
\hline & \multicolumn{2}{|r|}{1} & \multicolumn{2}{|r|}{2} & \multicolumn{2}{|r|}{$2 a$} & \multicolumn{2}{|r|}{$2 \mathrm{~b}$} \\
\hline & Mean & Range & Mean & Range & Mean & Range & Mean & Range \\
\hline \multicolumn{9}{|l|}{ SUB } \\
\hline \# Samples & & 3 & & 5 & & 2 & & 3 \\
\hline \# Persons & & 3 & & 3 & & 2 & & 2 \\
\hline$c_{\mathrm{f}}(\mathrm{g} / \mathrm{ml})$ & 0.15 & $0.12-0.18$ & 0.82 & $0.44-1.09$ & 1.02 & $0.94-1.09$ & 0.69 & $0.44-0.97$ \\
\hline$n_{\mathrm{f}}$ & 1.36 & $1.35-1.36$ & 1.47 & $1.41-1.52$ & 1.51 & $1.50-1.52$ & 1.45 & $1.41-1.49$ \\
\hline$d_{\mathrm{e}}(\AA)$ & 580 & $500-700$ & 2600 & $1100-3600$ & 1400 & $1100-1700$ & 3500 & $3300-3600$ \\
\hline$\Gamma_{\mathrm{e}}\left(\mathrm{mg} / \mathrm{m}^{2}\right)$ & 9 & $8.5-9.0$ & 200 & $120-340$ & 140 & $120-160$ & 240 & $160-340$ \\
\hline$t_{0}(\mathrm{~h})$ & 0.6 & $0.2-1$ & 4 & $3-5$ & 3.5 & $3-4$ & 4 & $4-5$ \\
\hline$t_{\mathrm{H}}(\min )$ & 1 & $0-2$ & 20 & $10-30$ & 25 & $24-26$ & 17 & $10-30$ \\
\hline$\Gamma_{e} / \tau\left(\mathrm{mg} / \mathrm{m}^{2} \mathrm{~h}\right)$ & 360 & $350-370$ & 470 & $210-760$ & 240 & $210-260$ & 630 & $460-760$ \\
\hline \multicolumn{9}{|l|}{ PAR } \\
\hline \# Samples & & 3 & & 4 & & & & \\
\hline \# Persons & & 3 & & 2 & & & & \\
\hline$c_{\mathrm{f}}(\mathrm{g} / \mathrm{ml})$ & 0.21 & $0.17-0.23$ & 0.66 & $0.31-1.00$ & & & & \\
\hline$n_{\mathrm{f}}$ & 1.37 & $1.36-1.37$ & 1.45 & $1.39-1.50$ & & & & \\
\hline$d_{\mathrm{e}}(\AA)$ & 480 & $400-600$ & 1500 & $1200-1700$ & & & & \\
\hline$\Gamma_{e}\left(\mathrm{mg} / \mathrm{m}^{2}\right)$ & 9.5 & $9.0-10$ & 93 & $53-120$ & & & & \\
\hline$t_{0}(\mathrm{~h})$ & 3 & $1.5-4$ & 3 & $2-4$ & & & & \\
\hline$t_{\mathrm{H}}(\mathrm{min})$ & 1 & $0-2$ & 15 & $12-18$ & & & & \\
\hline$\Gamma_{e} / \tau\left(\mathrm{mg} / \mathrm{m}^{2} \mathrm{~h}\right)$ & 330 & $200-420$ & 270 & $120-320$ & & & & \\
\hline \multicolumn{9}{|l|}{ WHL } \\
\hline \# Samples & & & & 7 & & & & \\
\hline \# Persons & & & & 5 & & & & \\
\hline$c_{\mathrm{f}}(\mathrm{g} / \mathrm{ml})$ & & & 1.13 & $1.05-1.19$ & & & & \\
\hline$n_{\mathrm{f}}$ & & & 1.52 & $1.51-1.53$ & & & & \\
\hline$d_{\mathrm{e}}(\AA)$ & & & 990 & $780-1600$ & & & & \\
\hline$\Gamma_{\mathrm{e}}\left(\mathrm{mg} / \mathrm{m}^{2}\right)$ & & & 113 & $89-190$ & & & & \\
\hline$t_{0}(\mathrm{~h})$ & & & 3.5 & $3-5$ & & & & \\
\hline$t_{\mathrm{H}}(\min )$ & & & 21 & $13-41$ & & & & \\
\hline$\Gamma_{\mathrm{e}} / \tau\left(\mathrm{mg} / \mathrm{m}^{2} \mathrm{~h}\right)$ & & & 240 & $180-320$ & & & & \\
\hline
\end{tabular}

Note. $\Gamma_{\mathrm{e}}$ is calculated using $n_{\mathrm{solv}}=1.3327$ and $d n / d c=0.17 \mathrm{ml} / \mathrm{g} . t_{0}$ is the time at which $\Gamma(t)$ is maximal. Other quantities are discussed in the text.

area, $\Gamma$, showed a time dependence similar to the curve in Fig. 5, with end values ranging from about 8 to $320 \mathrm{mg} / \mathrm{m}^{2}$ ( $\Gamma$ was calculated from Eq. [8] using $n_{\text {solv }}=1.3327$ (water) and $d n / d c=0.17 \mathrm{ml} / \mathrm{g}$ ). In a few cases a final equilibrium situation was not reached within 3 to $4 \mathrm{~h}$ : $\Gamma$ or $d$ (or both) appeared to increase with a constant rate of change.

\subsection{Bulk Measurements}

The measured bulk properties are summarized in Table 1. Note that the range in these properties for whole saliva samples is more limited than those for the other types of saliva. Furthermore the flow rate of secretion is given in the same table.

Of several samples the $\mathrm{pH}$ was measured both before and after the ellipsometric measurements. These $\mathrm{pH}$ measurements indicated only small changes ( $<0.1 \mathrm{pH}$ units) for several hours. The wide range of $\mathrm{pH}$ values is a biological variation, both between different persons and between different samples of one person. It is a well-known phenomenon that 


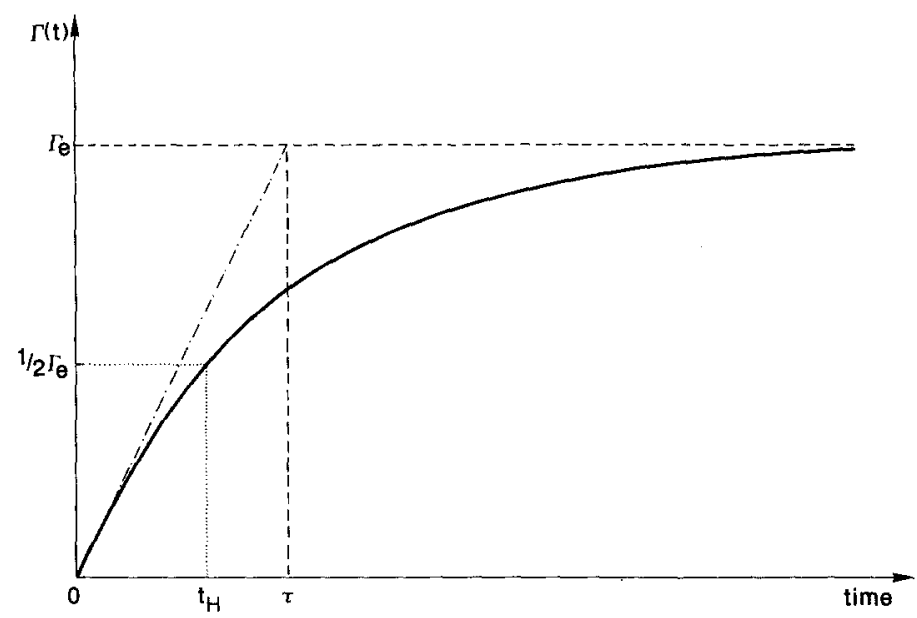

FIG. 6. Assumed exponential adsorption behavior.

a high flow rate corresponds to a high $\mathrm{pH}$ value fore and after the ellipsometric measurements of the sample (4). The same phenomenon was showed still clear solutions, without any preobserved in this study. The saliva samples be- cipitation.

\section{TABLE III}

Bulk Properties Classified Corresponding to the Classification of Table II

\begin{tabular}{|c|c|c|c|c|c|c|c|c|}
\hline & \multicolumn{2}{|r|}{1} & \multicolumn{2}{|r|}{2} & \multicolumn{2}{|r|}{$2 a$} & \multicolumn{2}{|r|}{$2 \mathrm{~b}$} \\
\hline & Mean & Range & Mean & Range & Mean & Range & Mean & Range \\
\hline \multicolumn{9}{|l|}{ SUB } \\
\hline \# Samples & & 3 & & 5 & & 2 & & 3 \\
\hline $\begin{array}{l}\text { Flow rate } \\
(\mathrm{ml} / \mathrm{min})\end{array}$ & 0.9 & $0.73-1.1$ & 0.85 & $0.63-1.1$ & 0.7 & $0.63-0.76$ & 0.9 & $0.64-1.1$ \\
\hline$n_{\mathrm{s}}$ & 1.3333 & $1.3330-1.3335$ & 1.3332 & $1.3330-1.3335$ & 1.3331 & $1.3330-1.3332$ & 1.3333 & $1.3331-1.3335$ \\
\hline$\rho\left(\mathrm{kg} / \mathrm{m}^{3}\right)$ & 999.3 & $998.8-999.5$ & 999.3 & $998.5-1000.1$ & 998.9 & $998.5-999.2$ & 999.6 & $998.7-1000.1$ \\
\hline $\mathrm{pH}$ & 6.9 & $6.8-7.1$ & 7.6 & $7.3-7.9$ & 7.5 & $7.3-7.6$ & 7.7 & $7.5-7.9$ \\
\hline$\Lambda\left(10^{-3} \Omega^{-1} \mathrm{~cm}^{-1}\right)$ & 3.2 & $2.4-3.6$ & 3.8 & $2.4-6.0$ & 3.0 & $2.4-3.6$ & 4.3 & $2.4-6.0$ \\
\hline \multicolumn{9}{|l|}{ PAR } \\
\hline \# Samples & & 3 & & 4 & & & & \\
\hline $\begin{array}{l}\text { Flow rate } \\
\qquad(\mathrm{ml} / \mathrm{min})\end{array}$ & 0.35 & $0.34-0.35$ & 1.5 & $1.1-1.9$ & & & & \\
\hline$n_{\mathrm{s}}$ & 1.3336 & $1.3333-1.3340$ & 1.3338 & $1.3338-1.3340$ & & & & \\
\hline$\rho\left(\mathrm{kg} / \mathrm{m}^{3}\right)$ & 1000.2 & $999.4-1000.9$ & 1000.7 & $998.9-1001.9$ & & & & \\
\hline $\mathrm{pH}$ & 6.4 & $5.1-7.4$ & 8.2 & $8.1-8.4$ & & & & \\
\hline$\Lambda\left(10^{-3} \Omega^{-1} \mathrm{~cm}^{-1}\right)$ & 3.5 & $3.0-3.8$ & 5.9 & $5.4-7.0$ & & & & \\
\hline \multicolumn{9}{|l|}{ WHL } \\
\hline \# Samples & & & & 7 & & & & \\
\hline \multicolumn{9}{|l|}{ Flow rate } \\
\hline$(\mathrm{ml} / \mathrm{min})$ & & & 0.74 & $0.28-0.9$ & & & & \\
\hline$n_{\mathrm{s}}$ & & & 1.3333 & $1.3332-1.3335$ & & & & \\
\hline$\rho\left(\mathrm{kg} / \mathrm{m}^{3}\right)$ & & & 999.3 & $999.0-1000.0$ & & & & \\
\hline $\mathrm{pH}$ & & & 7.5 & 7.4-7.7 & & & & \\
\hline$\Lambda\left(10^{-3} \Omega^{-1} \mathrm{~cm}^{-1}\right)$ & & & 3.3 & $2.9-4.0$ & & & & \\
\hline
\end{tabular}




\section{DISCUSSION}

On the basis of the type of saliva and the concentration in the surface layer a classification can be made (Table II). The concentration $c_{\mathrm{f}}$ in the surface layer is calculated from

$$
c_{\mathrm{f}}=\Gamma_{\mathrm{e}} / d_{\mathrm{e}}
$$

The samples of each type of saliva can be divided into two groups according to a low and high $c_{\mathrm{f}}$ value, respectively. It is striking that the classification with respect to $c_{\mathrm{f}}$ also implies a clear separation with respect to $d_{\mathrm{e}}$ and $\Gamma_{\mathrm{e}}$. Obviously the refractive index $n_{\mathrm{f}}$ also differs between different groups because $c_{\mathrm{f}}$ is directly related to $n_{\mathrm{f}}$ (as can be seen from Eqs. [8] and [10]).

Again the ranges for whole saliva samples are much smaller than for the other types of saliva. In addition no low concentration group exists at all. The wide range in measurement values for submandibular and parotid saliva may be partly due to variation in intensity of stimulation, while stimulation during whole saliva secretion was relatively low. In all cases, however, saliva surface layers are much thicker than the adsorption layers on several other protein solutions as described in the literature, while layer concentrations have similar values $(2,9)$.

The high concentration group of the submandibular samples (SUB2 group) can be subdivided with respect to $d_{\mathrm{e}}$.

The time $t_{\mathrm{H}}$ at which an amount $\Gamma_{\mathrm{e}} / 2$ per unit area has been adsorbed appears to be much shorter in the case of low $c_{\mathrm{f}}$ (and thus low $\Gamma_{\mathrm{e}}$ ) than in the case of high $c_{\mathrm{f}}$ (and thus high $\Gamma_{e}$ ). One may wonder whether there exists an empirical relationship between $t_{\mathrm{H}}$ and $\Gamma_{\mathrm{e}}$. The following rough approach attempts to quantify this point.

Langmuir's simple monolayer adsorption model results in an exponential time dependence (10). Assuming a similar dependence in a multilayer situation (which is more likely to occur in our case) we may write

$$
\Gamma(t)=\Gamma_{\mathrm{e}}\left(1-\exp \frac{-t}{\tau}\right)
$$

(see also Fig. 6).

In first approximation such a graph fits roughly experimentally. The adsorption rate $d \Gamma / d t$ at times much shorter than $\tau$ approaches $\Gamma_{\mathrm{e}} / \tau$ as can be derived from Eq. [11]. The half-way time $t_{\mathrm{H}}$ can be calculated by inserting $\Gamma\left(t_{\mathrm{H}}\right)=\Gamma_{\mathrm{e}} / 2$, resulting in $t_{\mathrm{H}}=\tau \ln 2$.

Therefore, the initial adsorption rate becomes

$$
\left.\frac{d \Gamma}{d t}\right|_{0}=\frac{\Gamma_{\mathrm{e}} \cdot \ln 2}{t_{\mathrm{H}}}
$$

Thus quantifying the experimental $\Gamma(t)$ curve in first approximation with the mentioned time dependence, the initial adsorption rate can be estimated from the determined $\Gamma_{\mathrm{e}}$ and $t_{\mathrm{H}}$ values.

The averaged value of $\Gamma_{e} / \tau$ over all samples is $330 \mathrm{mg} / \mathrm{m}^{2} \mathrm{~h}$ (standard error $150 \mathrm{mg} / \mathrm{m}^{2}$ $h)$. The averaged values in the various sample groups are given in the classification table (Table II).

Though the adsorption process behind the assumed time dependence (Eq. [11]) is in the present case a large oversimplification, it is interesting to observe that all $\Gamma_{\mathrm{e}} / \tau$ values are equal within approximately $50 \%$, suggesting that initially the layer formation in all cases is identical. Though this approach quite successfully unifies all the measurements with respect to the relationship between $t_{\mathrm{H}}$ and $\Gamma_{\mathrm{e}}$, scrutinizing the actual graph reveals that the experimental path is steeper at short times and less steep at long times than the graph from Fig. 6. A comparison to other protein solutions could not be made, since adsorption kinetic studies in the literature were all performed at much lower bulk concentrations. It is likely that the sample separation into different groups is caused by differences in the bulk quantities. Comparing Tables II and III reveals a clear correlation between bulk $\mathrm{pH}$ and surface layer parameters. 


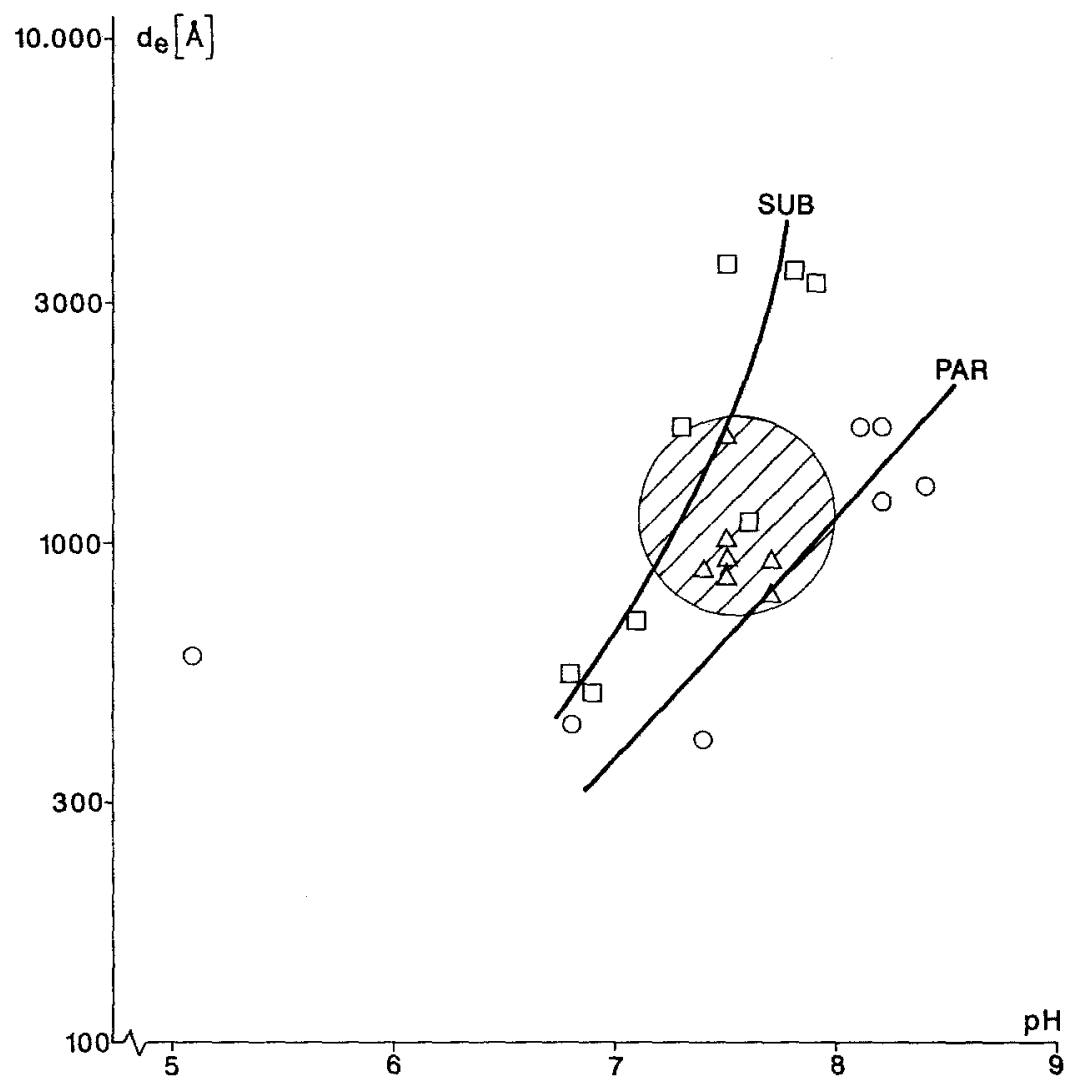

FIG. 7. Correlation between the thickness of the surface layer and the pH of the bulk liquid. ( $\square$, submandibular; $O$, parotid; $\Delta$, whole saliva. The hatched area represents the region of samples of whole saliva.)

As an example the correlation between bulk $\mathrm{pH}$ and layer thickness is shown in Fig. 7. It can be seen that even small changes in $\mathrm{pH}$ are correlated to large changes in layer thickness. The PAR2 group distinguishes itself from other groups because of its high $\mathrm{pH}$ value. It is noteworthy to mention that three samples within this group are from the same person.

\section{REFERENCES}

1. Waterman, H. A., 's-Gravenmade, E. J., and van der Wallen Mijnlieff, P. F., J. Dent. Res. 64, 716 (1985).

2. de Feyter, J. A., Benjamins, J., and Veer, F. A., Biopolymers 17, 1759 (1978).
3. Azzam, R. M. A., and Bashara, N. M., "Ellipsometry and Polarized Light." North-Holland, Amsterdam, 1977.

4. Mason, D. K., and Chisholm, D. M., "Salivary Glands in Health and Disease," Chap. 3. Saunders, London, 1975.

5. McCrackin, F. L., Passaglia, E., Stromberg, R. R., and Steinberg, H. L., J. Res. Natl. Bur. Stand. A 67, 363 (1963).

6. McCrackin, F. L., Natl. Bur. Stand. US Technical Note 479 (1969).

7. Schneyer, L. H., J. Dent. Res. 34, 257 (1955).

8. Lashley, K. S., J. Exp. Psychol. 1, 461 (1916).

9. Graham, D. E., and Phillips, M. C., J. Colloid Interface Sci. 70, 403, 415 (1979).

10. Langmuir, I., J. Amer. Chem. Soc. 40, 1361 (1918). 